\title{
Blastomycosis-like pyoderma in a case of chronic myeloid leukaemia
}

\author{
Tarun K. Dutta ${ }^{1}$, Joseph James ${ }^{1}$, M.C. Baruah ${ }^{2}$ and C. Ratnakar ${ }^{3}$ \\ Departments of ${ }^{1}$ Medicine, ${ }^{2}$ Dermatology and ${ }^{3}$ Pathology, JIPMER, Pondicherry 605 006, India
}

\begin{abstract}
Summary: Blastomycosis-like pyoderma, a rare skin lesion which may clinically resemble true blastomycosis, is seen in immuno-compromised individuals. We report one such case in chronic myeloid leukaemia.
\end{abstract}

\section{Introduction}

There are several non-specific skin lesions known to occur in association with myelogenous leukaemias. Commonest amongst them are acute febrile neutrophilic dermatosis (Sweet's syndrome) and atypical or superficial, bullous variant of pyoderma gangrenosum. ${ }^{1-3}$ We report a case of blastomycosis-like pyoderma, a rare skin lesion which may accompany chronic myeloid leukaemia. ${ }^{4}$ There have been earlier reports of blastomycosis-like pyoderma from India, ${ }^{5,6}$ but none associated with chronic myeloid leukaemia.

\section{Case report}

A 50 year old female, found to have chronic myeloid leukaemia one year previously was admitted with multiple skin lesions, which had appeared over the previous 3 weeks. She had been taking busulphan $4 \mathrm{mg}$ daily since diagnosis.

On examination, the patient was afebrile and had no significant lymphadenopathy. There were multiple verrucous plaques with an elevated border on the face, upper limbs and trunk (Figure 1), which we first assumed to be leukaemic infiltrates. She also had verrucous and ulcerated lesions on the back (Figure 2) and pustules over the neck. Most of the plaques and nodules were studded with pusfilled cavities particularly on the periphery. There was hepatosplenomegaly.

Investigations revealed haemoglobin $7.5 \mathrm{~g} / \mathrm{dl}$, white cell counts $21.2 \times 10^{9} / 1$, platelet $160 \times 10^{9} / 1$, blood differential count: neutro - $58 \%$, lympho $11 \%$, basophil $8 \%$, promyelocyte $3 \%$, myelocyte

Correspondence: Tarun K. Dutta, M.B., B.S., M.D. Accepted: 7 October 1991
$10 \%$, metamyelocyte and stab form $10 \%$. Blood karyotyping showed mosaic pattern with $25 \%$ cells with $46 \mathrm{XX}$ chromosomes and $75 \%$ cells with Philadelphia chromosome $\left(\mathrm{Ph}^{1}\right)$. Bone marrow report was suggestive of chronic myeloid leukaemia in a stable phase.

Smears from skin lesions were negative for acid-fast bacillus: Gram's stain showed Grampositive cocci in clusters suggesting Staphylococcus aureus. Potassium hydroxide preparation for fungal element was negative. Pus culture could not be done.

A diagnosis of blastomycosis-like pyoderma was considered, skin biopsy performed and the patient was put on co-trimoxazole, to which also staphylococci are generally sensitive ${ }^{7,8}$ (cloxacillin was not available in our hospital at that time). All the skin lesions healed within a week.

Multiple sections studied from skin biopsy showed pseudoepitheliomatous hyperplasia of the epidermis. There were multiple microabscesses in the hyperplastic epidermis as well as in the dermis. The abscesses were composed predominantly of neutrophils. Only occasional eosinophil and mononuclear cells were seen. A histological diagnosis of blastomycosis-like pyoderma was made (Figure 3).

\section{Discussion}

Blastomycosis-like pyoderma is a rare disorder more often seen in an immuno-compromised host, where the patient has an exaggerated immunological response to a bacterial infection such as with Staphylococcus. The immuno-compromised states likely to be present may be as wide as leukaemia, immunosuppressant therapy, X-ray radiation, pulmonary granuloma or diabetes mellitus. ${ }^{4,5} \mathrm{Su}$ et al. ${ }^{4}$ also observed this condition in chronic myeloid 


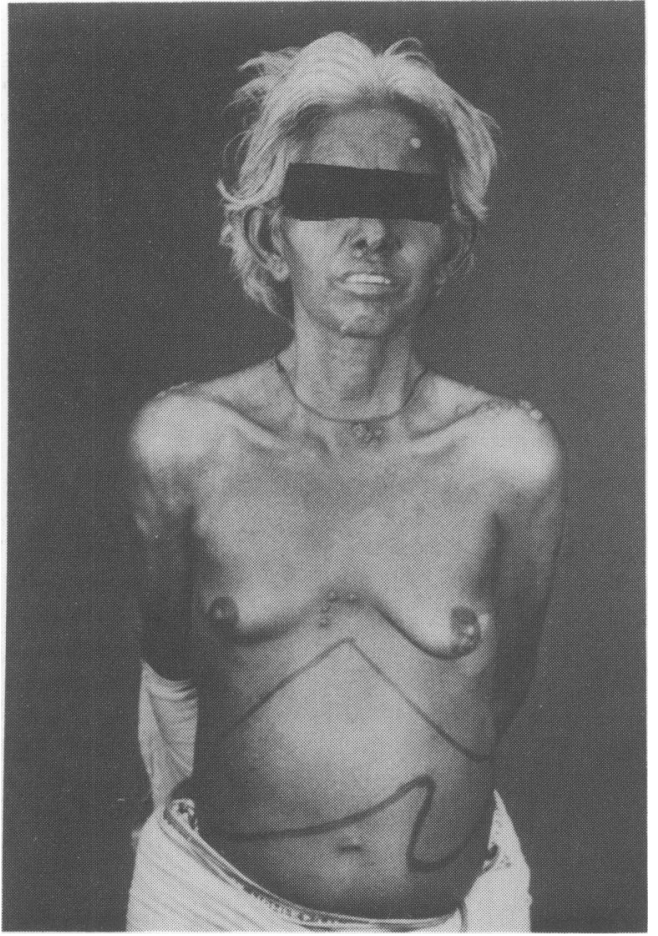

Figure 1 Clinical photograph showing multiple verrucous-plaques on the face, upper limbs and trunk. Hepatosplenomegaly is outlined.

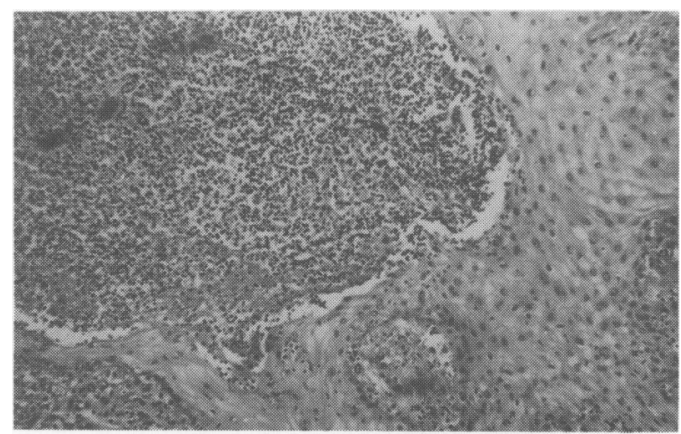

Figure 3 Photomicrograph showing pseudoepitheliomatous hyperplasia of the epidermis with multiple microabscesses in the dermis and epidermis (H\&E $\times 100)$.

leukaemia in one of their cases. However, the disease can occur in normal individuals as evidenced by the report of Kumar et al., ${ }^{6}$ where none out of seven cases had an underlying immuno-compromised disorder. Our patient was immuno-compromised due to her disease and the chemotherapy.

The condition is characterized by the appearance

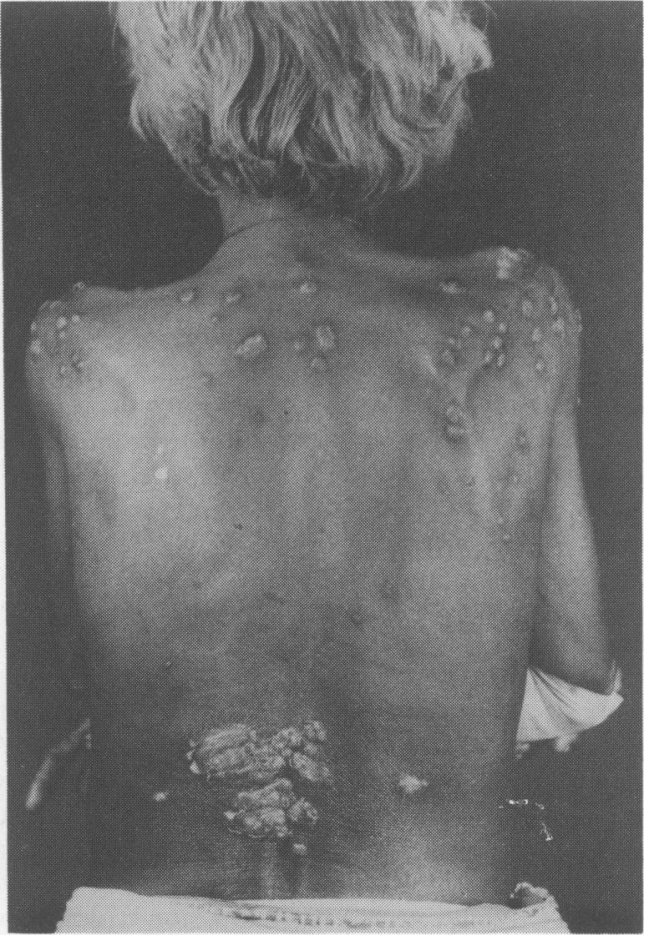

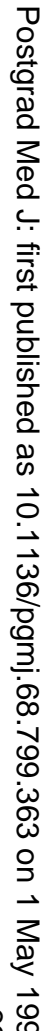

Figure 2 Verrucous and ulcerated lesions on the back

of large crusted verrucous plaques with multiple pustules and elevated border. ${ }^{4}$ The characteristic lesions occur either singly or in multiples and are located on the face, neck, forearm, hand, leg or foot. ${ }^{4}$

Our initial suspicion of leukaemic infiltrate appeared doubtful in view of the multiple pustular cavities. The picture also did not appear to be that of atypical pyoderma gangrenosum, because of its verrucoid appearance. At this stage, the diagnosis of blastomycosis-like pyoderma was considered. The lesions of blastomycosis-like pyoderma may clinically simulate true blastomyocosis or cutaneous tuberculosis. ${ }^{5}$ However, pus smear study was negative for acid-fast bacillus and fungal element in our patient. Further, these conditions have characteristic histopathology. In a country like India, where cutaneous tuberculosis is rampant, blastomycosis-like pyoderma ought to be an important differential diagnosis in patients with warty hyperkeratotic lesions. ${ }^{5}$

The typical histopathological feature in this disorder is the presence of pseudoepitheliomatous hyperplasia and multiple abscesses in dermis and epidermis with a neutrophilic reaction. In the initial stage, pseudoepitheliomatous hyperplasia of this condition may be confused with irregular prolifera- 
tion of squamous cells in squamous cell carcinoma. $^{4}$

$\mathrm{Su}$ et al.$^{4}$ laid down certain strict criteria for the diagnosis of blastomycosis-like pyoderma. They include large verrucous plaques with multiple pustules and elevated border, pseudoepitheliomatous hyperplasia with abscesses in tissue biopsy specimen, growth of at least one pathogenic bacterium,

\section{References}

1. Piette, W.W. An approach to cutaneous changes caused by hematologic malignancies. In: Callen, J.P. \& Jorizzo, J.L. (eds) Dermatologic Clinics: Skin Signs of Internal Disease. W.B. Saunders, Philadelphia, 1989, Vol. 7, No. 3, pp. 467-479.

2. Callen, J.P. Pyoderma gangrenosum and related disorders. In Callen, J.P., Dahl, M.V., Golitz, L.E., Schachner, L.A. \& Stegman, S.J. (ed.) Advances in Dermatology, Year Book Medical Publishers, Inc., Chicago, 1989, Vol. 4, pp.51-70.

3. Dover, J.S. \& Imber, M.J. A 47-year-old man with a rash, fever, and headaches (case records of the Massachusetts General Hospital). N Engl J Med 1990, 323: 254-263.

4. Su, W.P.D., Duncan, S.C. \& Perry, H.O. Blastomycosis-like pyoderma. Arch Dermatol 1979, 115: 170-173.

5. Singh, M., Kumar, B., Kaur, S. et al. Blastomycosis-like pyoderma. Ind J Dermatol Venereol Leprol 1985, 51: 226-228. such as $S$. aureus, $\beta$-haemolytic streptococci or Pseudomonas aeruginosa, negative culture for deep fungi, atypical mycobateria and Mycobacterium tuberculosis, negative fungal serology test and a normal bromide level in blood. Though our patient did not fulfil all the above-mentioned criteria, we arrived at a diagnosis of blastomycosis-like pyoderma after taking the total picture into account.

6. Kumar, V., Garg, B.R. \& Baruah, M.C. Blastomycosis-like pyoderma. Ind J Dermatol Venereol Leprol 1990, 56: 58-60.

7. Mandell, G.L. \& Sande, M.A. Anti-microbial agents: sulfonamides, trimethoprim - sulphamethoxazole, quinolones, and agents for urinary tract infections. In: Gilman, A.G., Rall, T.W., Nies, A.S. \& Taylor, P. (eds) Goodman and Gilman's The Pharmacological Basis of Therapeutics, 8th ed (International Edition). Maxwell Macmillan, Singapore, 1991, Vol. 2, pp. 1047-1064.

8. Sande, M.A., Kapusnik-Uner, J.E. \& Mandell, G.L. Antimicrobial agents: general considerations. In: Gilman, A.G. Rall, T.W., Nies, A.S., Taylor, P. (eds) Goodman and Gilman's The Pharmacological Basis of Therapeutics, 8th ed (International Edition). Maxwell Macmillan, Singapore, 1991, Vol. 2, pp. 1018-1046. 